\title{
Locality and Modular Invariance in 2D Conformal QFT*
}

\author{
K.-H. Rehren \\ Inst. f. Theor. Physik \\ Universität Göttingen \\ 37073 Göttingen \\ Germany \\ Dedicated to S. Doplicher and J.E. Roberts.
}

\begin{abstract}
The relations and differences between various classification problems arising in the context of local two-dimensional (2D) conformal quantum field theory, modular invariants, and subfactors, are discussed. The extent to which locality implies modular invariance, is exhibited.
\end{abstract}

AMS Subject classification: 81T40, 46L37 (primary); 81T05 (secondary)

\section{Introduction: Modular invariants, 2D conformal QFT, and subfactors}

One of the great excitements in conformal QFT was the ADE classification of modular invariant coupling matrices for the $\mathrm{SU}(2)$ current algebra [5]. At each level $k$, this algebra has only a finite number of covariant representations with positive energy (superselection sectors), and the partition functions for the conformal Hamiltonian in these sectors span a linear representation of the modular group $S L(2, \mathbb{Z})$ acting on the temperature parameter. A modular invariant is a quadratic form (described by the coupling matrix $Z$ ) in this space of partition functions which is invariant under the modular group, and which is subject to a number of additional constraints. These constraints are necessary for the modular invariant being interpretable as the partition function of a local 2D conformal QFT with a unique vacuum vector. This $2 \mathrm{D}$ conformal QFT contains the $\mathrm{SU}(2)$ current algebra both as left and right chiral subalgebras.

The fact that $S U(2)$ modular invariants can be classified according to an ADE scheme, has raised the interest of mathematicians both for its arithmetic appeal, and for its relationship to several other classification problems (for an overview see [27]). As a physicist,

${ }^{*}$ Contributed to "Mathematical Physics in Mathematics and Physics", Siena, June 20-25, 2000 
I want to focus on the quantum field theoretical aspect of this classification problem. I also want to consider it from a broader perspective, namely as a statement about the possible position of the embedding of two (left and right) chiral subtheories $A_{L}$ and $A_{R}$ into a local $2 \mathrm{D}$ conformal quantum field theory $B$. The case of $S U(2)$ is then just a very special one, and an exact ADE classification cannot be expected to prevail in general.

From this perspective, the classification problem is to find all local $2 \mathrm{D}$ conformal QFT's which extend the given chiral subtheories $A_{L}$ and $A_{R}$. These extensions are also (though not completely) characterized by a coupling matrix $Z$. The nontrivial requirement here which imposes constraints on $Z$, is locality, while modular invariance is a secondary feature.

Finally, there is a third aspect of modular invariants which is of interest for the classification of subfactors. The embedding of chiral subtheories into a local 2D conformal QFT gives rise to local subfactors of the form $A \otimes C \subset B$ which are called canonical tensor product subfactors 22. The specific meaning of "canonical" will be explained below, and is again related to the existence of a coupling matrix. Such subfactors also arise, e.g., as generalized quantum doubles or as asymptotic subfactors. A general classification is not available, but several results on canonical tensor product subfactors will be reported, which have direct implications with regard to the modular and local QFT classification problems.

I emphasize that in the local perspective, modular invariance is an additional requirement which is often imposed on the $2 \mathrm{D}$ conformal QFT by string theory demands. In fact, it is not independent from the requirement of locality. Several results below show that 2D locality "almost" implies modular invariance - but not quite, since there are easy examples of local 2D extension with a coupling matrix which is not modular invariant. On the other hand, among the modular invariant coupling matrices for affine Lie algebras of higher rank, there are some accidental ones, which do not come from any local 2D conformal QFT (e.g., [25]).

In order to further clarify the relation between locality and modular invariance, we recall the notion of "statistics characters" introduced in [20] which directly derives from locality. It provides a pair of matrices $X$ and $Y$ defined in terms of the statistics of a system of superselection sectors (Sect. 3). $X$ is the diagonal matrix of the statistics phases, while $Y$ collects the values of the (relative) monodromy operators in a natural tracial state. Unless there is some degeneracy in the statistics, these matrices yield another unitary representation of the modular group $S L(2, \mathbb{Z})$ ("statistics representation", Prop. 3.3). A priori, this representation is not related to modular transformations of the temperature parameter of the partition functions for these sectors. (Note also that partition functions need not even exist a priori as Gibbs functionals.) But at least for affine Lie algebras and certain related algebras, both representations of the modular group exist, and coincide.

The counter examples mentioned above (local 2D conformal QFT, but not modular invariant) are due to the possible non-coincidence of the two representations. In many cases, even if thermodynamic modular invariance fails, the analogous invariance property with respect to the matrices $X$ and $Y$ still holds true. I propose to call this invariance property "(degenerate or nondegenerate) statistics symmetry" (Def. 3.4). Nondegenerate 
statistics symmetry is equivalent to modular invariance with respect to the statistics representation (Prop. 3.5). Useful criteria for statistics symmetry will be provided (e.g., the maximal chiral observables within the $2 \mathrm{D}$ theory always have it), but there are also examples of local 2D theories which satisfy not even statistics symmetry, cf. Sect. 3.

I shall not discuss the characterization of solutions to the various classification problems in terms of graphs (such as ADE). Further details on this aspect can be found in [3, 19, 27].

\section{The modular invariants perspective}

One considers a rational chiral QFT $A$ and the finite system of its covariant representations $\pi_{i}$ with positive energy. The most prominent examples for $A$ are affine Lie algebras (current algebras), the Virasoro algebra with central charge $c<1$, or certain $W$-algebras arising in coset constructions. One assumes that the characters $\chi_{i}(\beta)$ for each of these representations exist as Gibbs functionals (i.e., $\exp -\beta \pi_{i}\left(L_{0}\right)$ are trace class operators), and that the characters transform linearly in a unitary representation of the $S L(2, \mathbb{Z})$ transformations of the complex temperature parameter $\tau=i \beta / 2 \pi$ in the regime $\operatorname{Im}(\tau)>0$ (and further parameters, if necessary). This assumption is fulfilled for all the examples mentioned [11].

The unitary matrix representatives for the generating transformations $\tau \rightarrow \tau+1$ and $\tau \rightarrow-1 / \tau$ are commonly called $T$ and $S$, respectively. The square of the latter transformation is a central involution in $S L(2, \mathbb{Z})$, and unity in $P S L(2, \mathbb{Z})$. Hence $C=S^{2}$ commutes with $S$ and $T$, and $C^{2}=\mathbb{1}$. The $S L(2, \mathbb{Z})$ relations are thus

$$
T S T S T=S, \quad C T=T C, \quad C S=S C=S^{-1} .
$$

One looks for modular invariant quadratic forms of the form

$$
\mathcal{Z}(\tau)=\sum_{i j} Z_{i j} \chi_{i}(\tau) \chi_{j}(\bar{\tau})
$$

with a coupling matrix $Z$, satisfying

$$
T Z=Z T \quad \text { and } \quad S Z=Z S \quad \text { (modular invariance) } .
$$

In addition one postulates that the matrix entries $Z_{i j}$ are nonnegative integers, and $Z_{00}=$ 1 (the label 0 is always reserved for the vacuum sector). These are necessary conditions if one wants to interpret $\mathcal{Z}$ as a partition function $\operatorname{Tr} \exp \left(-\operatorname{Re}(\beta) P_{\text {conf }}^{0}-i \operatorname{Im}(\beta) P_{\text {conf }}^{1}\right)$ where $P_{\text {conf }}^{\mu}$ are the conformal Hamiltonian and momentum of a $2 \mathrm{D}$ conformal QFT with a unique vacuum vector. $Z_{i j}$ then is the multiplicity of the product of chiral sectors $\pi_{i} \otimes \pi_{j}$ within the vacuum representation of the $2 \mathrm{D}$ theory. One considers

The modular classification problem: Find all coupling matrices $Z$ with nonnegative integer entries and $Z_{00}=1$, which commute with the given pair of matrices $S$ and $T$ (and find out and discard the accidental ones, which do not correspond to a local $2 \mathrm{D}$ theory, see above). 
One finds several types of solutions, which come in pairs because with $Z$ also $Z C=C Z$ is a solution. There is always the diagonal solution, $\mathcal{Z}=\sum_{i} \chi_{i} \otimes \chi_{i}$, along with the conjugate diagonal solution $\mathcal{Z}=\sum_{i} \chi_{i} \otimes \chi_{\bar{i}}$. All solutions share the "block form"

$$
\mathcal{Z}=\sum_{I} \chi_{I} \otimes \chi_{\sigma(I)}
$$

where the "extended characters" $\chi_{I}=\sum_{i} N_{i I} \chi_{i}$ unite certain "families" $I$ of sectors, and $\sigma$ is some permutation of the families. Among these, there are the orbifold solutions where sectors $i$ and $j$ belong to the same family if and only if they differ by a simple sector (with respect to fusion) belonging to some group of simple sectors. Solutions with the identical permutation are called Type I, those with a nontrivial permutation $\sigma$ are called Type II.

For the $S U(2)$ affine Lie algebra, it turns out that for all modular invariants, the nonvanishing diagonal entries $Z_{i i} \neq 0$ can be identified with the Coxeter exponents of a Dynkin diagram of type $\mathrm{A}, \mathrm{D}$ or $\mathrm{E}[5]$. The $A_{n}$ diagrams correspond to the diagonal type I invariants, $D_{\text {odd }}$ to permutation and $D_{\text {even }}$ to orbifold invariants, respectively. $E_{6}$ and $E_{8}$ correspond to the exceptional Type I, and $E_{7}$ to the exceptional Type II invariants. These ADE invariants exhaust all modular invariants for $S U(2)$. There are similar classifications for $S U(3)$, but an exact match with Dynkin diagrams cannot be achieved $[\$$ ].

\section{The local QFT perspective}

We consider

The local classification problem: Find all local 2D conformal QFT's which irreducibly extend the given pair of chiral theories $A=A_{L} \otimes A_{R}$,

$$
A_{L} \otimes A_{R} \subset B
$$

To formalize the problem, I adopt the algebraic framework of QFT; thus a QFT is given by a net of local von Neumann algebras (factors), say $A(I)$ with $I$ a light ray interval, or $B(O)$ with $O$ a $2 \mathrm{D}$ double-cone, subject to standard axioms including essential Haag duality. An inclusion like (2.1) is thus always understood as the collection of local subfactors $A_{L}(I) \otimes A_{R}(J) \subset B(O)$ for all $O=I \times J$.

There is no a priori reason from the local QFT perspective why the left and right chiral subtheories $A_{L}$ and $A_{R}$ of a $2 \mathrm{D}$ conformal QFT $B$ should be isomorphic (parity symmetric). I prefer to include the "heterotic" (non-symmetric) case in my discussion from the beginning.

The left and right chiral observables within the $2 \mathrm{D}$ conformal theory $B$ must commute with the respective opposite Möbius group. It has been shown [21] that all 2D observables which are invariant under the right Möbius group, indeed define a chiral subtheory, referred to as the maximal left chiral observables $A_{L}^{\max }$. These contain the given subtheory of left chiral observables $A_{L}$, and one has the intermediate inclusion

$$
A_{L} \otimes A_{R} \subset A_{L}^{\max } \otimes A_{R}^{\max } \subset B .
$$


Anticipating results below, one finds that the maximal chiral observables coincide with the "extended observables" in the modular invariants context [16], with respect to which the coupling matrix turns into a permutation matrix thus giving rise to the block form (1.4). This fact is due, in view of Prop. 4.1 below, to the following equivalent characterization of the maximal chiral observables [21].

Proposition 2.1 21] Let $B$ be a local 2D conformal QFT, and $A_{L}, A_{R}$ chiral subtheories whose observables generate the left and right Möbius groups, respectively. For any doublecone region of conformal $2 D$ Minkowski space, $O=I \times J$ in light ray coordinates, one has

$$
A_{L}(I) \subset A_{L}^{\max }(I)=B(O) \cap U\left(G_{R}\right)^{\prime}=B(O) \cap A_{R}(J)^{\prime} .
$$

(The corresponding staments hold for $R \leftrightarrow L$.) In particular, $A_{L}^{\max }(I)$ and $A_{R}^{\max }(J)$ are each other's relative commutants in $B(O)$.

The subalgebras of left and right chiral observables form an algebraic tensor product, and at least in the cyclic subspace of the vacuum they are also represented as a tensor product [21]. It is expected that the tensor product is spatial in the full representation of the 2D theory, and we shall assume this in the sequel.

This means that the vacuum representation of $B$, as a representation of the subalgebra $A_{L} \otimes A_{R}$ decomposes into irreducibles according to the scheme

$$
\pi \simeq \bigoplus_{i j} Z_{i j} \pi_{i}^{L} \otimes \pi_{j}^{R}
$$

The coupling matrix $Z$ appearing in this decomposition serves as a first (though not complete) characterization of the extension. Again, $Z$ has nonnegative integer entries, and $Z_{00}=1$ because of the uniqueness of the vacuum vector, but the labels $i$ and $j$ may run over different sets (the sectors of $A_{L}$ and of $A_{R}$ ), and $Z$ may be rectangular. Although a priori the direct sum might be countably or even uncountably infinite, we shall assume it to be finite throughout.

It follows that the thermodynamical partition function of the $2 \mathrm{D}$ conformal $\mathrm{QFT}$, if it exists, is of the most general form in terms of chiral Gibbs functionals,

$$
\mathcal{Z}=\sum_{i j} Z_{i j} \chi_{i}^{L}(\tau) \chi_{j}^{R}(\bar{\tau})
$$

with the same coupling matrix $Z$ as in the decomposition (2.4). The notions of "coupling matrix" thus coincide in the modular and in the local interpretation. I emphasize, however, that coupling matrices associated with local 2D extensions are not necessarily modular invariant. This issue will be discussed in Sect. 3.

Due to the intermediate inclusion (2.2) the coupling matrix is a product

$$
Z=B_{L}^{t} Z^{\max } B_{R}
$$

where $Z^{\max }$ is the coupling matrix with respect to the maximal left and right chiral observables, and the branching matrices $B_{L}$ and $B_{R}$ describe the irreducible decomposition 
of the chiral superselection sectors $\pi_{I}^{\max }$ of $A^{\max }$ upon restriction to $A \subset A^{\max }$. The restricted representations $\left.\pi_{I}^{\max }\right|_{A} \simeq \bigoplus B_{I i} \pi_{i}$ play a role like the families of sectors in eq. (1.4). This analogy will also be exhibited in Sect. 3.

The question arises by which data of the chiral subtheories, apart from the coupling matrix, their local 2D extensions can be characterized. (It should be stressed that the coupling matrix will in general not determine the local extension up to equivalence, although it happens to do so in models with "few" sectors.) An answer has been given in 114 for the more general problem of characterizing local extensions $B$ of a QFT $A$ in any dimension.

The local extension $B$ of a quantum field theory $A$ is characterized by its canonical DHR triple $\left(\rho, w, w_{1}\right)$ (assuming the index $\lambda$ of the local subfactors $A(O) \subset B(O)$ to be finite) [14]. Here, $\rho$ is the DHR endomorphism [6] of $A$ which describes the vacuum representation of $B$ as a reducible representation of $A$, and the isometric intertwiners $w: \operatorname{id}_{A} \rightarrow \rho, w_{1}: \rho \rightarrow \rho^{2}$ in $A$ satisfy the identities of a "Q-system" [13]

$$
\begin{gathered}
w^{*} w_{1}=\rho\left(w^{*}\right) w_{1}=\lambda^{-\frac{1}{2}} \mathbb{1}_{A}, \\
w_{1} w_{1}=\rho\left(w_{1}\right) w_{1}, \quad w_{1} w_{1}^{*}=\rho\left(w_{1}^{*}\right) w_{1},
\end{gathered}
$$

and the eigenvalue condition for the statistics operator [6] $\varepsilon(\rho, \rho) \in \rho^{2}(A)^{\prime}$

$$
\varepsilon(\rho, \rho) w_{1}=w_{1}
$$

The significance of the operator identities (2.7) and (2.8) has been explained in 23]: The sector of $\rho$ describes the DHR charges [6] with respect to the subtheory $A$ which appear in the vacuum representation of $B$. The isometry $w$ singles out the vacuum (charge zero) sector within $\rho$, and $w_{1}$ is a generating functional for the collection of $3 j$-symbols (amplitudes of 3-point functions of charge carrying fields). The identities (2.7) reflect the condition that the corresponding linear combinations of localized vertex operators (charged field bundle elements [7]) form a $*$ algebra. The eigenvalue condition (2.8) expresses the condition that these combinations, which constitute the fields of $B$, commute at spacelike distance.

The extension $B$ is recovered from the canonical DHR triple as follows [14]. If $\rho$ is localized in some space-time double-cone $O_{0}$, then it restricts to an endomorphism of the corresponding local algebra, $\rho_{0} \in \operatorname{End}\left(A\left(O_{0}\right)\right)$, and $\left(\rho_{0}, w, w_{1}\right)$ is a Q-system which determines the local algebra $B\left(O_{0}\right)$ extending $A\left(O_{0}\right)$. With the help of unitary charge transporters for $\rho$, the local Q-system $\left(\rho_{0}, w, w_{1}\right)$ can be moved to every Poincaré or Möbius transformed double-cone $O=g\left(O_{0}\right)$, giving rise to a coherent net of algebras $B(O)$ extending $A(O)$. These satisfy local commutativity thanks to (2.8).

We conclude that local extensions of local QFT's are completely characterized by canonical DHR triples, that is, solutions $\left(\rho, w, w_{1}\right)$ to $(2.7)$ and $(2.8)$ within the category of DHR endomorphisms and their intertwiners. For rational QFT's, these operator identities constitute a finite system of nonlinear algebraic equations which may serve as the basis of the present classification problem. 
In the $2 \mathrm{D}$ case of our interest, $A=A_{L} \otimes A_{R}$ is a tensor product, and so is its DHR category. The endomorphism $\rho$ entering the problem is given by eq. (2.4),

$$
\rho \simeq \bigoplus_{i j} Z_{i j} \alpha_{i}^{L} \otimes \alpha_{j}^{R}
$$

Its specification is equivalent to the specification of the coupling matrix. Thus, the local classification problem is equivalent to

The local classification problem (algebraic version): Find all canonical DHR triples for the given pair of chiral theories $A=A_{L} \otimes A_{R}$ with $\rho$ of the form (2.9).

The local classification problem involves the determination of all admissible coupling matrices. As was mentioned before and will be discussed in more detail in the next section, these do not coincide with the modular invariant coupling matrices. Nevertheless, the question arises whether for any given modular invariant, an associated local 2D conformal QFT exists, and whether this will be unique. We therefore address also

The existence and uniqueness problem for local 2D conformal QFT: Decide whether a given coupling matrix $Z$ for $A_{L} \otimes A_{R}$ (modular invariant or not) arises as the coupling matrix of a local 2D conformal QFT, and whether the latter is unique.

There is no reason to expect that the uniqueness problem will have a positive answer in general. It is well known that non-isomorphic subfactors can have the same canonical endomorphism (hence the same coupling matrix). The eigenvalue condition (2.8) will presumably not alter the situation very much.

As for the existence problem, some progress has been made. A standard solution of (2.7) and (2.8) has been given in [14 for the coupling matrix $Z=C$ pertaining to the parity symmetric case $A_{L} \simeq A_{R}$, based on Prop. 4.2 below. This means that conjugate left and right chiral DHR charges can always be combined to yield local $2 \mathrm{D}$ fields. The standard solution exists for any closed (under fusion and conjugation) subsystem of DHR sectors of $A$, and thus yields truncated conjugate diagonal coupling matrices which are typically not modular invariant.

A more general class of possibly heterotic solutions can be obtained from Prop. 4.3 below for all $S U(2)$ and many other modular invariant coupling matrices.

\section{On the relation between modular invariance and locality}

It is appropriate to discuss the distinction between the local and the modular classification problem.

First, it is not completely understood in which sense the Gibbs partition functions of any chiral theory $A$ should transform linearly under the modular group in general when no Kac-Peterson formula [11] is available. (Higher rank current algebras already exhibit pairs of conjugate sectors with the same partition function, and this degeneracy has to be lifted by introducing additional thermodynamic parameters on which the modular group can act.) The most general result in this direction is due to Nahm [18. Only the subgroup generated by $T$ acts in general, which measures the spectrum of $L_{0}$ modulo integers. 
Second, one should not exclude heterotic models with different left and right chiral observables, as such models might arise upon passage to "extended chiral algebras" [16], even if one starts with parity symmetric models. The left and right chiral algebras then might have different modular transformation matrices, and the requirement of modular invariance is no longer a commutation property, but an intertwining property:

$$
T_{L} Z=Z T_{R} \quad \text { and } \quad S_{L} Z=Z S_{R} \quad \text { (heterotic modular invariance). }
$$

If we enlarge the framework accordingly, the question arises to which extent modular invariance of the coupling matrix of a local 2D extension is implied by the properties of a canonical DHR triple.

The intertwining property for $T$ is easily established. According to the standard argument, all Wightman fields affiliated with the local 2D theory $B$ must be Bose fields, hence should have integer difference between left and right chiral scaling dimensions. In the algebraic framework, this follows from the eigenvalue condition:

Lemma 3.1 Let $\left(\rho, w, w_{1}\right)$ be a canonical DHR triple (in any dimension), and $\sigma$ an irreducible subsector of $\rho$. Then $\sigma$ has statistics phase +1 .

Proof: The statistics parameter of $\rho$ [6]

$$
\phi_{\rho}(\varepsilon(\rho, \rho))=r^{*} \rho(\varepsilon(\rho, \rho)) r=\rho\left(r^{*}\right) \varepsilon(\rho, \rho)^{*} r
$$

can be computed: inserting the isometry $r=w_{1} w: \mathrm{id}_{A} \rightarrow \rho^{2}$, and using (2.8) and (2.7) yields

$$
\phi_{\rho}(\varepsilon(\rho, \rho))=\rho\left(w^{*} w_{1}^{*}\right) w_{1} w=\rho\left(w^{*}\right) w_{1} w_{1}^{*} w=\lambda^{-1} \mathbb{1}=d(\rho)^{-1} \mathbb{1} .
$$

Since the spectrum of the statistics parameter determines the statistics phases of the subsectors [6, 7], the claim follows.

Q.E.D.

Corollary 3.2 In the $2 D$ conformal case, with $A=A_{L} \otimes A_{R}$, let $\sigma \prec \rho$ be of the tensor product form $\sigma_{L} \otimes \sigma_{R}$. Then $\sigma_{L}$ and $\sigma_{R}$ have equal statistics phases. Hence the coupling matrix intertwines the left and right diagonal matrices $X$ of statistics phases,

$$
X_{L} Z=Z X_{R}
$$

Proof: Spacelike separation in 2D means positive left separation and negative right separation, or vice versa. Hence the statistics operator of $\sigma$ is the tensor product of two opposite chiral statistics operators, and the statistics phase of $\sigma$ is the quotient of the chiral statistics phases. From this, the statements are obvious.

Q.E.D.

Now, the chiral statistics phases $\kappa$ are related to the chiral scaling dimensions $h$ by the spin-statistics theorem [9], $\kappa=\exp 2 \pi i h$. Thus, the matrices $T$ differ from the matrices $X$ by an overall complex phase $\exp 2 \pi i \frac{c}{24}$ depending on the chiral central charges 11 . It follows that the coupling matrix $Z$ intertwines $T_{L}$ and $T_{R}$ up to the quotient of these 
phases. Thus, locality always implies invariance under the modular transformation $T$ up to a phase which is trivial if the left and right central charges coincide modulo 24 .

A statement of comparable generality cannot be obtained for the full modular group. E.g., the local $2 \mathrm{D}$ theory $B=A_{L} \otimes A_{R}$ has the coupling matrix $Z_{i j}=\delta_{i 0} \delta_{j 0}$ which is definitely not modular invariant. But we can obtain nontrivial general results concerning a symmetry which specializes to modular invariance in favorable cases. As this symmetry does not refer to Gibbs functionals but to statistics, I call it "statistics symmetry" (Def. 3.4). We shall see that it is always satisfied, as a consequence of locality, for the maximal chiral observables.

Thus, rather than the thermodynamical transformation matrices $T$ and $S$, we consider the statistics phase matrix $X$ (the same as in Cor. 3.2) and the statistics character matrix $Y$, defined in [20]. These matrices can be written as

$$
X_{i j}=X_{j i}=\kappa_{i} \delta_{i j} \quad \text { and } \quad Y_{i j}=Y_{j i}=\sum_{k} N_{i j}^{k} \frac{\kappa_{i} \kappa_{j}}{\kappa_{k}} d_{k}
$$

where $i, j, k$ run over all irreducible DHR sectors of the model (in the present case: of the chiral observables $A_{L}$ or $A_{R}$ ), or over any closed subsystem $\Delta$ thereof. $\kappa_{i}$ and $d_{i}$ are the complex phase and the inverse modulus of the statistics parameter [6, 7] of the sector $\pi_{i}$, and $N_{i j}^{k}$ are the fusion rules. For a non-exhaustive system $\Delta$, the matrices $X$ and $Y$ are truncated to those rows and columns of the full matrices which correspond to $\Delta$.

Proposition 3.3 20 The matrices $X$ and $Y$ satisfy the relations

$$
X Y X Y X=z Y, \quad C X=X C, \quad C Y=Y C=Y^{*}
$$

where $z=\sum_{i \in \Delta} \kappa_{i} d_{i}^{2}$ and $C$ is the charge conjugation matrix. If $Y$ is invertible, a rescaling of $X$ and $Y$ yields unitary matrices $T^{\text {stat }}$ and $S^{\text {stat }}$ which satisfy (1.1) and hence generate a representation ("statistics representation") of $S L(2, \mathbb{Z})$, turning $\Delta$ into a modular category [17].

(More specifically, in the nondegenerate case, $|z|^{2}$ equals the "global index" $w=$ $\sum_{i \in \Delta} d_{i}^{2}$ of the system of sectors, and $\left.S^{\text {stat }}=w^{-1 / 2} Y, T^{\text {stat }}=(z /|z|)^{-1 / 3} X\right)$.

It is expected that $T^{\text {stat }}=T$ and $S^{\text {stat }}=S$, whenever modular transformation matrices $T$ and $S$ exist independently. In fact, the first of these coincidences is essentially the spinstatistics theorem [9] which relates the statistics phase determining $T^{\text {stat }}$ to the chiral scaling dimension ("spin") entering $T$. The second one is at least empirically true wherever it has been tested.

That $Y$ is indeed invertible provided $\Delta$ is the system of all DHR sectors, was recently shown [12] to follow from the split property, and is therefore true whenever the chiral Gibbs functionals $\operatorname{Tr} \exp -\beta \pi_{i}\left(L_{0}\right)$ exist for any temperature $1 / \beta>0$.

Let us now turn to the question of statistics symmetry.

Definition 3.4 A local 2D extension satisfies statistics symmetry if

$$
X_{L} Z=Z X_{R} \quad \text { and } \quad \frac{1}{\lambda_{L}} Y_{L} Z=\frac{1}{\lambda_{R}} Z Y_{R} \quad \text { (statistics symmetry). }
$$


The factors $\lambda_{L}=\sum_{i} Z_{i 0} d\left(\pi_{i}^{L}\right)$ (and likewise $\left.\lambda_{R}\right)$ in the $Y$ relation are dictated by comparison of the 00 components. They equal the indices $\lambda=d\left(\left.\pi_{0}^{\max }\right|_{A}\right)$ of the inclusions $A \subset A^{\max }$ (because $\left(B_{R}\right)_{J 0}=\delta_{J 0}$ and $Z_{I 0}^{\max }=\delta_{I 0}$, hence $\left.Z_{i 0}=\left(B_{L}\right)_{0 i}\right)$.

Proposition 3.5 If $Y_{L}$ and $Y_{R}$ are nondegenerate, statistics symmetry implies

$$
T_{L}^{\text {stat }} Z=Z T_{R}^{\text {stat }} \quad \text { and } \quad S_{L}^{\text {stat }} Z=Z S_{R}^{\text {stat }} \quad \text { (nondegenerate statistics symmetry), }
$$

i.e., the coupling matrix is a modular invariant with respect to the left and right statistics representations of $S L(2, \mathbb{Z})$ (cf. Prop. 3.3).

Proof: The claim is clearly true up to scalar factors. Eqs. (3.4) and (3.5) imply $z_{L} / \lambda_{L}=$ $z_{R} / \lambda_{R}$. The complex phase and the modulus of this equality imply that all scalar factors cancel. (Incidentally, [⿴囗十, Prop. 3.1] implies that the left and right global indices $w_{L}=w_{R}$ and the inclusion indices $\lambda_{L}=\lambda_{R}$ coincide separately.)

Q.E.D.

Cor. 3.2 states that the $X$ part of statistics symmetry follows directly from the locality condition on the 2D extension. Locality also enters the proof of the $Y$ part, but suitable completeness conditions will be required in addition, cf. Cor. 3.8.

In order to discuss the intertwining relation for $Y$, we recall from Sect. 2 the product form of the total coupling matrix

$$
Z=B_{L}^{t} Z^{\max } B_{R}
$$

due to the intermediate inclusion

$$
A_{L} \otimes A_{R} \subset A_{L}^{\max } \otimes A_{R}^{\max } \subset B
$$

Here $Z^{\max }$ is the coupling matrix for the maximal observables, and $B_{L}$ and $B_{R}$ are the branching matrices. From Prop. 2.1 we know that the maximal chiral observables are each other's relative commutants, and in fact are distingushed by this property. Prop. 4.1 below will tell us that the sets of sectors of $A_{L}^{\max }$ and $A_{R}^{\max }$ contributing to the coupling matrix (2.6) are both closed under fusion, and $Z^{\max }$ is a permutation matrix which induces an isomorphism of the fusion rules, and hence preserves the statistical dimensions. Since it also respects the statistics phases (Cor. 3.2), it intertwines the left and right statistics characters $Y^{\max }$ as well. In particular, $Y_{L}^{\max }$ is invertible if $Y_{R}^{\max }$ is, and the coupling matrix intertwines the ensuing left and right statistics representations.

Corollary 3.6 The coupling matrix of a local 2D conformal QFT with respect to its maximal chiral observables (or equivalently: every coupling matrix which is a permutation matrix) satisfies

$$
Y_{L}^{\max } Z^{\max }=Z^{\max } Y_{R}^{\max },
$$

hence statistics symmetry. If in addition both the left and right chiral statistics is nondegenerate, then the coupling matrix is a modular invariant with respect to the left and right statistics representations of $S L(2, \mathbb{Z})$. 
In [2] conditions were found that the branching matrices intertwine the matrices $Y$ and $Y^{\max }$ up to a numerical factor This implies that the total coupling matrix $Z=B_{L}^{t} Z^{\max } B_{R}$ also interwines $Y_{L}$ with $Y_{R}$, i.e., $Z$ has statistics symmetry:

Proposition 3.7 [9, Lemma 6.3] Consider a local extension (of chiral QFT's) $A \subset A^{\max }$ with finite index $\lambda$. If $\Delta$ is a closed system of DHR sectors of $A$, then its image $\alpha^{0}(\Delta)$ under ambichiral induction is a closed system of DHR sectors of $A^{\mathrm{max}}$, and the branching matrix $B$ intertwines the matrices $Y$ and $Y^{\max }$ associated with the systems $\Delta$ and $\alpha^{0}(\Delta)$ :

$$
\frac{1}{\lambda} B Y=Y^{\max } B
$$

"Ambichiral induction" is a map between closed systems of DHR endomorphisms, based on $\alpha$-induction. The latter is the natural prescription to extend DHR endomorphisms of a subtheory to an ambient theory, giving rise to soliton-type sectors in general. $\alpha$-induction was first considered by J. Roberts 24] in a cohomological problem, and its relevance for the local extension problem was discussed in [14]. Its functorial properties where first elaborated by Xu [26] and by Böckenhauer and Evans [1]. Due to nontrivial monodromies, there are in fact two prescriptions for $\alpha$-induction, and ambichiral induction is the intersection of the two sets of irreducible subsectors obtained by applying both prescriptions to the elements of $\Delta$.

An entry of the branching matrix $B$ is by definition the multiplicity of an irreducible DHR sector of $A$ in the restriction of an irreducible DHR sector of $A^{\text {max }}$. By " $\alpha-\sigma$ reciprocity" which holds for local extensions [1], this multiplicity equals the multiplicity of the DHR sector of $A^{\max }$ in the image of the sector of $A$ under either $\alpha$-induction. In particular, every DHR sector of $A^{\text {max }}$ belongs to the ambichiral image of each sector of $A$ contained in its restriction.

Corollary 3.8 Consider the local $2 D$ extension $B$ with coupling matrices $Z$ and $Z^{\max }$ with respect to the chiral observables $A_{L} \otimes A_{R}$ and $A_{L}^{\max } \otimes A_{R}^{\max }$, respectively. Let $\Delta_{X}$ and $\Delta_{X}^{\max }(X=L, R)$ be the sets of chiral DHR sectors contributing to the respective coupling matrices. If $\Delta_{X}$ are both closed systems, and $\Delta_{X}^{\max }$ both coincide with the images of $\Delta_{X}$ under ambichiral induction (or, equivalently, with the preimage of $\Delta_{X}$ under restriction), then the coupling matrix $Z$ satisfies statistics symmetry.

Proof: The equivalence of the two conditions on $\Delta_{X}^{\max }$ follows from $\alpha$ - $\sigma$-reciprocity and the obvious fact that $\Delta_{X}$ is the image of $\Delta_{X}^{\max }$ under restriction. According to Prop. 3.7, the branching matrices intertwine the statistics characters for the closed systems of sectors $\Delta_{X}$ and $\alpha^{0}\left(\Delta_{X}\right)$, while according to Cor. 3.6, the permutation matrix $Z^{\max }$ intertwines the statistics characters for the closed systems $\Delta_{L}^{\max }$ and $\Delta_{R}^{\max }$. Thus, under the stated conditions, Cor. 3.6 and Prop. 3.7 show that the coupling matrix $Z=B_{L}^{t} Z^{\max } B_{R}$ intertwines the statistics characters for $\Delta_{L}$ and $\Delta_{R}$ with the stated numerical factors.

Q.E.D.

This corollary is the strongest result one may expect for general heterotic theories, as a generalization of modular invariance implied by 2D locality. However, the conditions on 
the behavior of sectors under induction and restriction in the corollary (in order to apply the results of [2]) are not always satisfied.

Simple counter examples of perfectly local 2D extensions which violate the assumption and conclusion of Cor. 3.8 are given by

$$
A_{L} \otimes A_{R} \subset A_{L}^{\max } \otimes A_{R}^{\max }=B
$$

where the chiral inclusions $A \subset A^{\text {max }}$ have subfactor depth larger than 2, such as the "conformal embedding" $S U(2)_{10} \subset S p(5)_{1}$ with partition function

$$
\mathcal{Z}=\chi_{0}^{\max } \otimes \chi_{0}^{\max }=\left(\chi_{0}+\chi_{3}\right) \otimes\left(\chi_{0}+\chi_{3}\right) .
$$

Only those sectors of $A$ contribute to the coupling matrix which are contained in the vacuum sector of $A^{\max }$, and these do not form a closed system unless the depth is 2 . $\alpha$-induction on these sectors produces new DHR sectors of $A^{\text {max }}$ which are not contained in the vacuum representation of $B$. Completing the systems of chiral sectors ensures the correct intertwining property for the branching matrices, but the coupling matrix $Z^{\max }$, now being a bijection between subsystems of the completed systems, no longer intertwines $Y_{L}^{\max }$ with $Y_{R}^{\max }$ for the completed systems.

I do not see, whether the coupling matrix satisfies any sensible intertwining property weaker than statistics symmetry in complete generality. A possible criterium to exclude models like the counter examples (3.10), and hopefully to enforce the intertwining property (3.5), could be that the local 2D theory $B$ does not possess nontrivial superselection sectors, but I have no proof that this condition indeed has the desired consequences.

Thus, we see that 2D locality comes close to imply the statistics symmetry (Def. 3.4) which generalizes modular invariance to general heterotic models and to models without proper modular transformation laws for the chiral Gibbs functionals. But counter examples are easily constructed.

Conversely, as mentioned in the introduction, there are accidental modular invariants which do not admit a corresponding local 2D extension of the chiral observables. Thus, we conclude that modular invariance and 2D locality are intimately related while neither can imply the other without suitable further input.

\section{Canonical tensor product subfactors}

Solving the operator identities (2.7) within the DHR category of the local net $A$ is equivalent to finding a Q-system $\left(\rho_{0}, w, w_{1}\right)$ for the local factor $A\left(O_{0}\right)$ such that $\rho_{0} \in$ $\operatorname{End}_{\text {fin }}\left(A\left(O_{0}\right)\right)$ is the restriction of some DHR endomorphism of $A$ localized in $O_{0}$. The problem is thus of the type: Find all subfactors $A_{1}$ of a factor $A$ whose canonical endomorphisms $\rho$ decompose into irreducibles from a given system of sectors of $A$. In this form it is mathematically well-posed with any purely infinite factor $A$ and any given system of sectors $\Delta \subset \operatorname{End}_{\text {fin }}(A)$. To impose also the eigenvalue condition (2.8), one has to assume the system of sectors to be braided.

There is no general solution to this problem. But there are some partial results which relate to the specific tensor product structure. We call a subfactor of the form $A \otimes C \subset B$ 
a canonical tensor product subfactor (CTPS) [22] if its dual canonical endomorphism $\rho \in \operatorname{End}_{\text {fin }}(A \otimes C)$ decomposes into irreducibles as

$$
\rho \simeq \bigoplus_{i j} Z_{i j} \alpha_{i} \otimes \gamma_{j}
$$

with $\alpha_{i} \in \operatorname{End}(A)$ and $\gamma_{j} \in \operatorname{End}(C)$.

The embeddings $A_{L} \otimes A_{R} \subset B$ of Sect. 3 share this structure. The coupling matrix $Z$ of a CTPS is again in general rectangular. It has nonnegative integer entries, and $Z_{00}=1$ if and only if the CTPS is irreducible. We shall consider

The subfactor classification problem: Find all irreducible canonical tensor product subfactors $A \otimes C \subset B$ with $\alpha_{i}$ and $\gamma_{j}$ in (4.1) belonging to given systems of sectors of the given factors $A$ and $C$, respectively.

We have seen in Sect. 2 that the left and right maximal chiral observables are each other's relative commutants within the $2 \mathrm{D}$ theory. Therefore, the following general result, formulated in the broader framework of subfactors, is of interest.

Proposition 4.1 [21] Let $A \otimes C \subset B$ be a CTPS with dual canonical endomorphism as in (4.1). Then the following are equivalent.

(i) $\mathbb{1} \otimes C$ is the relative commutant of $A \otimes \mathbb{1}$ in $B$, and vice versa. (This property is called "normality").

(ii) $Z_{0 j}=\delta_{0 j}$ and $Z_{i 0}=\delta_{i 0}$.

(iii) The sectors $\alpha_{i}$ of $A$ and the sectors $\gamma_{j}$ of $C$ contributing to (4.1) are both closed under conjugation and fusion. There is a bijection between them which preserves the fusion rules, and the coupling matrix is the permutation matrix for this bijection.

It is not clear whether every CTPS $A \otimes C \subset B$ has an intermediate normal CTPS $\hat{A} \otimes \hat{C} \subset B$ as in Prop. 4.1. Presumably, this is not the case in general. The results in 21] on the tensor product position of chiral observables within a 2D conformal QFT, however, ensure the existence of a unique normal intermediate subfactor, corresponding to the maximal observables,

$$
A_{L} \otimes A_{R} \subset A_{L}^{\max } \otimes A_{R}^{\max } \subset B .
$$

The proposition, when applied to canonical DHR triples, thus splits the local classification problem into two independent parts: classification of chiral local extensions $A \subset A^{\text {max }}$, and classification of normal canonical DHR triples with $Z^{\text {max }}$ an isomorphism of the fusion rules of $A_{L}^{\max }$ and $A_{R}^{\max }$.

The proposition also implies that the coupling matrix (2.6) for a local $2 \mathrm{D}$ extension is of block form (1.4) as for modular invariants. (Note that in the heterotic case a distinction between "Type I" and "Type II" is meaningless.)

The CTPS classification problem can again be formulated algebraically in terms of Qsystems for $A \otimes C$ satisfying (2.7) with $\rho$ of the form (4.1). We cannot solve this problem systematically, but there are systematic prescriptions for the construction of Q-systems for CTPS's. In this way, the following Props. 4.2 and 4.3 were obtained, which are mainly relevant for the associated existence problem. 
Proposition 4.2 [14] Let $A$ be a purely infinite factor, and $\Delta \subset \operatorname{End}_{\text {fin }}(A)$ a finite closed system of irreducible endomorphisms $\sigma$ with finite index. Then there is a subfactor $A \otimes A^{\mathrm{opp}} \subset B$ with dual canonical endomorphism

$$
\rho \simeq \bigoplus_{\sigma \in \Delta} \sigma \otimes \sigma^{\mathrm{opp}}
$$

If the system $\Delta$ is braided, then the eigenvalue condition (2.8) is also satisfied.

The result has been generalized to factors of type II and to infinite systems $\Delta$ by Masuda [15], who also showed that the resulting subfactor is isomorphic to the asymptotic subfactor associated with the inclusion $\rho_{\Delta}(A) \subset A$ where $\rho_{\Delta}$ is the direct sum of all $\alpha_{i} \in \Delta$. Izumi has shown how to compute the complete structure of these subfactors [10].

The coupling matrix in Prop. 4.2 is the diagonal unit matrix for the system $\Delta$. In chiral conformal QFT on the circle, $A^{\text {opp }}=A(I)^{\text {opp }}$ is naturally isomorphic to $A(I)^{\prime}=A\left(I^{\prime}\right)$, and the latter is Möbius conjugate to $A(I)=A$. Under these identifications, the opposite $\sigma^{\text {opp }}$ of a DHR endomorphism $\sigma$ belongs to the charge conjugate sector $\bar{\sigma}$ [9]. Hence, in CQFT eq. (4.2) corresponds to a canonical DHR triple for $A_{L} \simeq A_{R}$ with coupling matrix $Z=C$, the charge conjugation matrix.

Applied to the existence problem for a given coupling matrix, or modular invariant, the proposition thus states that the conjugate diagonal matrix $Z=C$ always corresponds to a local 2D theory. By choosing irreducible subfactors $A_{\nu} \subset A(\nu=1,2)$, one obtains an abundance of CTPS's $A_{1} \otimes A_{2}^{\text {opp }} \subset B$ with coupling matrix in block form as with Type I modular invariants. The corresponding families of sectors of $A_{\nu}$ (cf. Sect. 1) are the restrictions of $\sigma \in \operatorname{End}_{\mathrm{DHR}}(A)$ to $A_{\nu}$.

The following result is an important generalization, by which local 2D conformal QFT's can be associated with numerous other (also Type II) modular invariants.

Proposition 4.3 29] Let $A_{\nu} \subset M(\nu=1,2)$ be two subfactors of finite index, $\Delta_{\nu} \subset$ $\operatorname{End}_{\text {fin }}\left(A_{\nu}\right)$ two finite closed systems of (inequivalent irreducible) endomorphisms. For a pair of inductions $\widehat{\cdot}$ (see below) of $\Delta_{\nu}$ put

$$
Z_{\sigma, \tau}=\operatorname{dim} \operatorname{Hom}(\widehat{\sigma}, \widehat{\tau})_{M} \quad\left(\sigma \in \Delta_{1}, \tau \in \Delta_{2}\right) .
$$

Then there is a CTPS $A_{1} \otimes A_{2}^{\text {opp }} \subset B$ with dual canonical endomorphism

$$
\rho \simeq \bigoplus_{\sigma \in \Delta_{1}, \tau \in \Delta_{2}} Z_{\sigma, \tau} \sigma \otimes \tau^{\mathrm{opp}}
$$

(An "induction" $\widehat{\cdot}$ assigns to each $\sigma \in \Delta$ an endomorphism $\widehat{\sigma} \in \operatorname{End}_{\text {fin }}(M)$ in a functorial manner: $\widehat{\sigma}$ extends $\sigma$, i.e., $\left.\widehat{\sigma}\right|_{A}=\sigma$, and intertwiners between products of $\sigma_{i}$ are intertwiners for products of $\widehat{\sigma}_{i}$ as well, i.e., $\operatorname{Hom}\left(\sigma_{1}, \sigma_{2} \sigma_{3}\right)_{A} \subset \operatorname{Hom}\left(\widehat{\sigma}_{1}, \widehat{\sigma}_{2} \widehat{\sigma}_{3}\right)_{M}$.)

If furthermore the systems $\Delta_{\nu}$ are braided, and the braidings fulfill a naturality condition with respect to Hom $(\widehat{\sigma}, \widehat{\tau})_{M}$, then the CTPS also satifies the eigenvalue condition (2.8). 
Let $A_{1} \simeq A_{2}$ be local algebras of chiral observables, $\Delta_{1} \simeq \Delta_{2}$ subsystems of their DHR endomorphisms, and assume that the dual canonical endomorphisms for the subfactors $A_{\nu} \subset M$ are also DHR endomorphisms (these subfactors need not describe local chiral extensions). Then $\alpha$-induction (cf. Sect. 3) is applicable to $\Delta_{\nu}$, and the assumptions of Prop. 4.3 are fulfilled with the two opposite prescriptions for $\alpha$-induction. It was shown in [4] that the corresponding coupling matrix (4.3) has statistics symmetry. In fact, many thermodynamical modular invariants can be obtained this way. For these modular invariants, Prop. 4.3 thus proves the existence of an associated local 2D conformal QFT.

The statement of Prop. 4.3 is, however, not restricted to $\alpha$-induction, and therefore produces a larger class of CTPS's than appearing in 2D conformal QFT. On the other hand, uniqueness of the subfactors in Props. 4.2 and 4.3 is not claimed. There may well be inequivalent CTPS's with the same dual canonical endomorphism, but with inequivalent Q-systems.

\section{Conclusions}

I have discussed the interrelations between several classification problems arising in mathematics and physics with various applications and interpretations: modular invariants, local 2D conformal QFT, and canonical tensor product subfactors. They all have some aspects in common (notably a coupling matrix), but the specific requirements imposed on the coupling matrix depend on the perspective. Progress in any of these classification problems will have a bearing on the related ones.

\section{Acknowledgments}

I thank J. Böckenhauer, S. Carpi and A. Ocneanu for numerous discussions, and J. Mund for a critical reading of the manuscript.

\section{References}

[1] Böckenhauer, J., Evans, D.E.: Modular invariants, graphs and $\alpha$-induction for nets of subfactors. I, Commun. Math. Phys. 197, 361-386 (1998), II, ibid. 200, 57-103 (1999), and III, ibid. 205, 183-229 (1999).

[2] Böckenhauer, J., Evans, D.E.: Modular invariants from subfactors: Type I coupling matrices and intermediate subfactors. math.OA/9911239.

[3] Böckenhauer, J., Evans, D.E.: Modular invariants from subfactors. math.OA/0006114.

[4] Böckenhauer, J., Evans, D.E., Kawahigashi, Y.: On $\alpha$-induction, chiral generators and modular invariants for subfactors. Commun. Math. Phys. 208, 429-487 (1999).

[5] Cappelli, A., Itzykson, C., Zuber, J.B.: The A-D-E classification of minimal and $A_{1}^{(1)}$ conformal invariant theories. Commun. Math. Phys. 113, 1-26 (1987).

[6] Doplicher, S., Haag, R., Roberts, J.E.: Local observables and particle statistics. I, Commun. Math. Phys. 23, 199-230 (1971), and II, ibid. 35, 49-85 (1974). 
[7] Fredenhagen, K., Rehren, K.-H., Schroer, B.: Superselection sectors with braid group statistics and exchange algebras. I, Commun. Math. Phys. 125, 201-226 (1989), and II, Rev. Math. Phys. SI1 (Special Issue), 113-157 (1992).

[8] Gannon, T.: The classification of SU(3) modular invariants revisited. Ann. Inst. H. Poinc. 65, 15-56 (1996).

[9] Guido, D., Longo, R.: The conformal spin and statistics theorem, Commun. Math. Phys. 181, 11-36 (1996).

[10] Izumi, M.: The structure of sectors associated with the Longo-Rehren inclusions. I, to appear in Commun. Math. Phys., and II, Kyoto preprint (2000).

[11] Kac, V.G., Peterson, D.H.: Infinite-dimensional Lie algebras, theta functions and modular forms. Adv. Math. 53, 125-264 (1984).

[12] Kawahigashi, Y., Longo, R., Müger, M.: Multi-interval subfactors and modularity of representations in conformal field theory. math.OA/9903104.

[13] Longo, R.: A duality for Hopf algebras and for subfactors. I, Commun. Math. Phys. 159, 133-150 (1994).

[14] Longo, R., Rehren, K.-H.: Nets of subfactors. Rev. Math. Phys. 7, 567-597 (1995).

[15] Masuda, T.: An analogue of Longo's canonical endomorphism for bimodule theory and its application to asymptotic inclusions. Int. J. Math. 8, 249-265 (1997).

[16] Moore, G., Seiberg, N.: Naturality in conformal field theory. Nucl. Phys. B313, 16-40 (1989).

[17] Müger, M.: Conformal field theory and Doplicher Roberts reconstruction. Contribution to this conference, math-ph/0008027.

[18] Nahm, W.: A proof of modular invariance. Int. J. Mod. Phys. A6, 2837-2845 (1991).

[19] Ocneanu, A.: Contribution to this conference.

[20] Rehren, K.-H.: Braid group statistics and their superselection rules. In: The Algebraic Theory of Superselection Sectors, D. Kastler (ed.). World Scientific, Singapore 1990, pp. 333-355.

[21] Rehren, K.-H.: Chiral observables and modular invariants. Commun. Math. Phys. 208, 689-712 (2000).

[22] Rehren, K.-H.: Canonical tensor product subfactors. Commun. Math. Phys. 211, 395-406 (2000).

[23] Rehren, K.-H., Stanev, Ya.S., Todorov, I.T.: Characterizing invariants for local extensions of current algebras. Commun. Math. Phys. 174, 605-633 (1996).

[24] Roberts, J.E.: Local cohomology and superselection structure. Commun. Math. Phys. 51, 107-119 (1976).

[25] Schellekens, A.N., Yankielowicz, S.: Field identification fix points in the coset construction. Nucl. Phys. B334, 67-102 (1990).

[26] Xu, F.: New braided endomorphisms from conformal inclusions. Commun. Math. Phys. 192, 349-403 (1998).

[27] Zuber, J.-B.: CFT, BCFT, ADE and all that. hep-th/0006151. 